\title{
A NEW TRANSFORMED STOCHASTIC SHORTEST PATH WITH DEAD ENDS AND ENERGY CONSTRAINT
}

\author{
Abdelhadi Larach ${ }^{1}$ and Cherki Daoui ${ }^{2}$ \\ Laboratory of Information Processing and Decision Support, \\ Sultan Moulay Slimane University, Faculty of sciences and Techniques, \\ Beni-Mellal, Morocco \\ ${ }^{1}$ larachabdelhadi@gmail.com, ${ }^{2}$ daouic@yahoo.com
}

\begin{abstract}
We consider Stochastic Shortest Path (SSP) Markov Decision Processes (MDPs) with dead ends and energy constraint. The objective is to find an optimal policy that maximizes the probability of reaching the target and minimizes the expected cost if the energy is sufficient. Firstly, we present a new Transformed SSP MDP that guarantees the convergence of the classical iterative algorithms and addresses the problem of energy-reachability. Secondly, we propose a topological algorithm based on a decomposition of the state space into some levels. In each level, a restricted SSP MDP is constructed and solved; the combined solution gives an admissible heuristic solution used as an initial point in the Pre-Gauss-Seidel Value Iteration (PGSVI) algorithm. Finally, an example of robot navigation in hexagonal grid's environment will be presented to show the advantages of the TSSP MDP and the performance of the proposed topological solver.
\end{abstract}

Keywords - Markov Decision Process, Graph theory, Stochastic Shortest Path, Value iteration, Decomposition technique

\section{INTRODUCTION}

The MDP theory has proven tremendously useful in a wide area of disciplines [1] and has received great attention from the Artificial Intelligence (AI) community as it offers easy frame work for modelling several complex real-life problems: robot navigation, Area Coverage [2], Vehicle routing problem, Stochastic Shortest Path (SSP) Problem, stochastic game playing [3], etc. Efficient solvers for MDPs has been developed; the most widely algorithms are Policy Iteration (PI), Value Iteration (VI) and its variants [4]. A SSP [5] is an MDP problem with positive costs and absorbing goal states. The objective is to find a strategy that reaches the goal or nearest goal state with lowest expected cost. If the state space contains unavoidable dead ends, the agent cannot always reach the goal with $100 \%$ probability. In this case, the SSP problem becomes multi-objective criterion: maximizing the probability to reach the goal and minimizing the expected cost.

Bonet and Geffner [6]-[8] propose some algorithms to solve SSP using undiscounted cost MDPs: i) a Real-Time Dynamic Programming (RTDP) [6], which is an approximation algorithm computing a heuristic solution by performing successive trials on the state space. RTDP outperforms VI in some domains; ii) Labelled RTDP (LRTDP) [7], which converges faster than RTDP because each state converged is labelled or marked as solved; iii) FIND-and-REVISE heuristic search framework [8], which computes the heuristic optimal cost vector from a given initial state without updating or

Received: May 8, 2019

Reviewed: July 26, 2019

Accepted: August 11, 2019

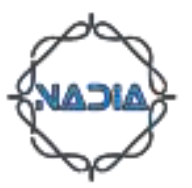


even memorizing cost for many of the other states . However, these solutions require that the goal must be reachable with $100 \%$ probability from any initial state, which cannot guarantee a solution with dead end states and does not deal with the insufficient resource case (energy-reachability). Kolobov et al., [9] propose an approach known as MAXPROB, which maximizes only the probability to reach the goal, as well as, a generalized class of SSP that allows more general reward structure; Weld et al., [10] present some approaches: $i$ ) SSP with Avoidable Dead Ends (SSPADE), which is a class of general SSP satisfying the avoidable condition to dead ends from a given initial state; ii) finite or infinite penalty for a visited Unavoidable Dead End (fSSPUDE, iSSPUDE), they use VI algorithm and Find-and-REVISE heuristic search framework [8]. Teichteil-Konigsbuch [11] and Kolobov et al., [12] propose other approaches by using a transformed SSP in which dead ends are removed and the probability of reaching the goal is maximized. Trevizan et al., [13] propose a new optimization criterion for SSPs known as Min-Cost given Max-Prob (MCMP criterion) based on a linear programming algorithm that is unfortunately expensive in time complexity especially, for a large state space.

In the main contribution of this work, the authors propose a new Transformed SSP MDP with dead ends that guarantees the convergence of the classical iterative algorithms and also addresses the energy-reachability problem. This transformation permits depending on three added parameters-to find an $\varepsilon$-optimal policy that: (1) surely avoid reaching the dead end state; (2) minimizes the expect cost if the energy is sufficient; (3) maximizes the probability of reaching the goal and minimizes the expected cost.

The remainder of this paper is organized as follows. Section 2 introduces some preliminaries on undiscounted MDPs and their iterative solvers. The proposed transformed SSP MDPs will be presented with its theoretical study in section 3 . Section 4 presents the topological solver. Simulations example of the TSSP MDP for robot navigation and experimental results are given in Section 5. In Section 6, we discuss the results and finally, we conclude and we present our perspectives in future works.

\section{BACKGROUND}

Markov Decision processes are defined as controlled stochastic processes satisfying the Markov property and assigning reward or cost values to state transitions [4],[14]. Formally, an MDP is defined by the five-tuple $(S, A, T, P, C)$, where: $S$ is the state space in which the process's evolution takes place; $A$ is the set of all possible actions which control the state dynamics; $T$ is the set of time steps where decisions need to be made; $P$ denotes the state transition probability function where $P_{i a j}=P\left(S_{t+l}=j \mid S_{t}=i, A_{t}=a\right)$ is the probability of transitioning to a state $j$ when an action $a$ is executed in a state $i, S_{t}\left(A_{t}\right)$ is a variable indicating the state (action) at time $t$; $C$ provides the cost function defined on state transitions where $C_{i a j}$ denotes the cost obtained if the action $a$ is applied in state $i$ and the process transits to state $j$. A pure strategy $\pi: S \rightarrow A$ is a decision rule. The core problem of MDPs is to find an optimal (or $\varepsilon$-optimal) strategy that specifies which action should be taken in each state. We denote by $F_{D}$ the set of pure strategies.

\subsection{UNDISCOUNTED COST MDP}

In the undiscounted infinite-horizon cost MDP, the value function, which is the expected cost when the process starts with state $i$ and using the policy $\pi$ is defined by:

$V_{\pi}(i)=E_{\pi}\left(\sum_{t=1}^{\infty} C_{x_{t} a_{t}}\right), i \in S$,

where $C_{x_{t} a_{t}}$ designs the cost obtained when the process is in state $x_{t}$ after $t$ steps and the action taken is $a_{t}$. When the cost value depends on the next state, it is defined by: 
$C_{x_{t} a_{t}}=\sum_{x_{t+1} \in S} P_{x_{t} a_{t} x_{t+1}} C_{x_{t} a_{t} x_{t+1}}$

where $C_{x_{t} a_{t} x_{t+1}}$ designs the cost obtained when the action $a_{t}$ is taken in state $x_{t}$, and the process transit to state $x_{t+1}$.

The objective is to determine $V^{*}$, the minimum expected total undiscounted cost vector over an infinite horizon defined by:

$$
V^{*}(i)=\operatorname{Inf}_{\pi \in F_{D}} V_{\pi}(i), i \in S
$$

It is well known that the minimum expected total undiscounted cost vector $V^{*}$ is the unique fixed-point of the following Bellman optimality equation [4], [14]:

$$
V(i)=\min _{a \in A(i)}\left\{\sum_{j \in S} P_{i a j}\left(C_{i a j}+V(j)\right)\right\}, i \in S
$$

Note that the existence of the fixed-point $V^{*}$ cannot be guaranteed for all MDP.

The actions attaining the minimum in Eq. 4 give rise to an optimal policy $\pi^{*}$ given by:

$$
\pi^{*}(i) \in \underset{a \in A(i)}{\operatorname{argmin}}\left\{\sum_{j \in S} P_{i a j}\left(C_{i a j}+V^{*}(j)\right)\right\}, i \in S
$$

\subsection{PRE-GAUSS-SEIDEL VALUE ITERATION SOLVER}

Value Iteration algorithm is one of the most widely standard iterative methods used to find an $\boldsymbol{\varepsilon}$-optimal policy for undiscounted MDPs [4, 14]. Starting with an initial cost vector $V^{0}$, the bellman backup operator is iteratively applied until convergence and, the optimal policy is given by Eq. 5. VI algorithm is executed in $O\left(\left.\|A\||\times| S\right|^{2}\right)$ arithmetic operations per iteration, where $\|A\|$ designs the average number of actions per state and $|S|$ the number of states. PGSVI algorithm is a variant of VI algorithm used in order to reduce the number of iterations and the memory consumption [4, 14]. In fact and since each value of the state updated is immediately replaced, only one vector is stored in the memory (lines 6 and 8, algorithm 1).

Algorithm 1: PGSVI Algorithm.

$$
\begin{aligned}
& \text { PGSVI(In: MDP: } \left.\mathrm{S}, \mathrm{P}, \mathrm{A}, \mathrm{C}, \varepsilon ; \mathrm{V}^{0} ; \text { Out: } \mathrm{V}^{*}, \pi^{*}\right) \\
& 1: \mathrm{V}^{*} \leftarrow \mathrm{V}^{0} \\
& \text { 2: Convergence } \leftarrow \text { False; //Convergence parameter } \\
& \text { 3: While ( not Convergence) Do //Iterative backup until convergence } \\
& \text { 4: } \text { Convergence } \leftarrow \text { True } \\
& \text { 5: For all i } \in \text { S Do //Bellman backup operator for each state } \\
& \text { 6: } \quad \mathrm{V}_{t m p} \leftarrow \min _{\mathrm{a} \in \mathrm{A}(\mathrm{i})}\left\{\sum_{j \in S} P_{i a j}\left(C_{i a j}+V^{*}(j)\right)\right\} \\
& \text { 7: } \quad \text { If }\left(\mathrm{V}_{t m p}-\mathrm{V}^{*}(\mathrm{i}) \mid \geq \varepsilon\right) \text { Convergence } \leftarrow \text { False } \\
& \text { 8: } \quad \mathrm{V}^{*}(\mathrm{i}) \leftarrow \mathrm{V}_{\text {tmp }} / / \text { Value immediately replaced } \\
& \text { 9: For each i } \in \text { S Do //Optimal policy given by Equation } 5 \\
& \text { 10: } \quad \pi^{*}(\mathrm{i}) \leftarrow \underset{\mathrm{a} \in \mathrm{A}(\mathrm{i})}{\operatorname{argmin}}\left\{\sum_{j \in S} P_{i a j}\left(C_{i a j}+V^{*}(j)\right)\right\}
\end{aligned}
$$

11: Return $V^{*}, \pi^{*}$ 


\section{STOCHASTIC SHORTEST PATH MDP}

A SSP MDP is defined by the tuple $\left(S, A, T, P, C, G, S_{0}\right)$, where:

- $\quad(S, A, T, P, C)$ are the MDP tuple.

- $G$ is a set of absorbing goal states, $\forall g \in G, P_{g a g}=1$ and $C_{g a}=0$ for all $a \in A$.

- $S_{0}$ is a set of a given start states.

The convergence of the iterative solvers such as VI or PGVI algorithms cannot be guaranteed for all SSP MDPs, especially when the state space contains unreachable targets from some initial states. To overcome this problem and to respond to the energyreachability, we present the following new Transformed SSP (TSSP) MDP.

\subsection{TRANSFORMED SSP MDP}

The TSSP MDP is defined by the tuple $\left(S^{\prime}, A^{\prime}, T, P^{\prime}, C^{\prime}, G^{\prime}, S_{0}, E, C_{d}, C_{a}, C_{g}\right)$ where:

- $S^{\prime}=\left(S \backslash S_{D}\right) \cup\left\{s_{d}\right\}$, where $S_{D}$ is the set of dead end states and $s_{d}$ is a single aggregated state representing $S_{D}$.

- $G^{\prime}=G \cup\left\{g^{*}\right\}$, where $g^{*}$ is an abstract goal state.

- $A^{\prime}=A \cup\{\theta\}, \theta$ is a fictitious action that moves the process to the abstract goal state $\mathrm{g}^{*}$, for all $s_{t} \in S^{\prime} \backslash\left\{s_{d}\right\}, A^{\prime}\left(s_{t}\right)=A\left(s_{t}\right) \cup\{\theta\}$.

- $\quad P^{\prime}{ }_{s_{t}} a_{t} s_{t+1}=P_{s_{t}} a_{t} s_{t+1}$ for all $a \in \mathrm{A}^{\prime}\left(s_{t}\right) \backslash\{\theta\}$ and $P^{\prime}{ }_{s_{t}} \theta g^{*}=1$

- $C^{\prime}{ }_{s_{t}} a_{t} s_{t+1}=C_{s_{t}} a_{t} s_{t+1}$ if $s_{t+1} \in S^{\prime} \backslash\left\{s_{d}\right\}$

- $\quad C^{\prime}{ }_{s t} \theta g^{*}=C_{a}$, where $C_{a} \geq 0$ designs the abstract positive cost incurred when the abstract goal state is reached.

- $C_{s_{t} a_{t} g}^{\prime}=C_{s_{t} a_{t} g}-C_{g}$, where $C_{g} \geq 0$ designs the reward added when the goal state is reached.

- $\quad C^{\prime}{ }_{s_{t} a_{t} s_{d}}=C_{s_{t} a_{t} s_{d}}+C_{d}$, where $C_{d} \geq 0$ designs the cost added when the dead end state is reached.

- $S_{0}$ is a set of a given start states.

- $\quad E$ designs the maximum cost supported by the agent to reach the goal state (initial energy).

Figure 1 presents an example of Transformed SSP MDP with one action in each state; black values design the transition probability and red values design the cost incurred; Dead end states $d_{1}$ and $d_{2}$ are aggregated to a single state $S_{d}$ and the action $\theta$ (red arrow) that moves to the abstract goal state $g *$ is added to all states with a cost value equal to $\mathrm{C}_{a}$; A cost value $C_{d}$ is added when transition to a dead end state is occurred; A reward value $\mathrm{C}_{\mathrm{g}}$ is added when transition to a goal state is observed. 


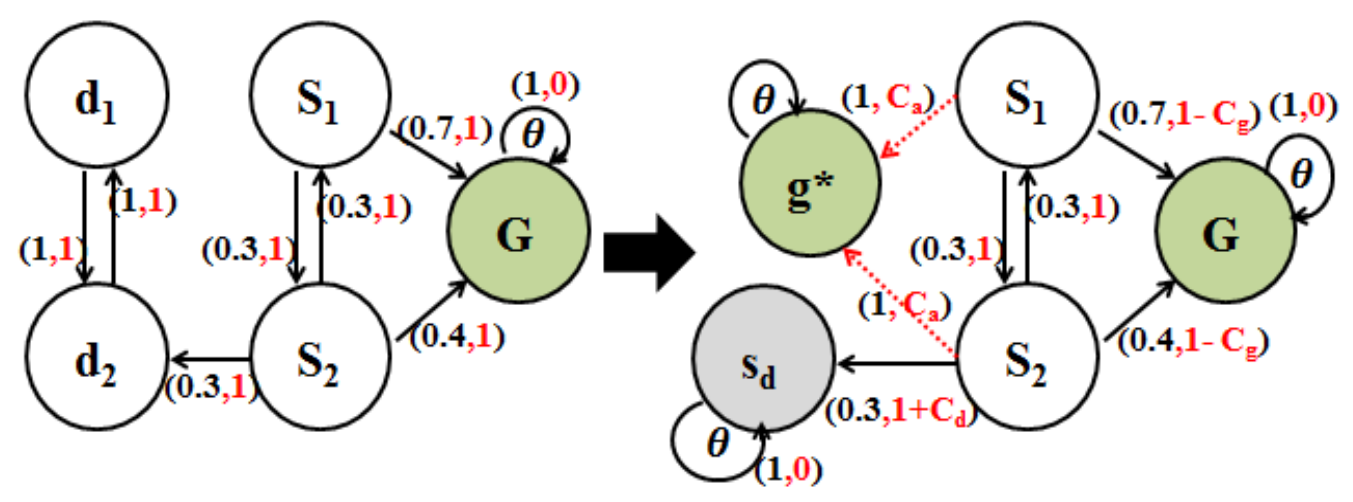

Fig. 1 Example of Transformed SSP MDP

\subsection{THEORETICAL STUDY}

Proposition 1: For the TSSP MDP, the expected total minimum cost vector $V^{*}$ is finite and bounded in the range $\left[-C_{g}, C_{a}\right]$.

Proof: Suppose that $V^{*}>C_{a}$ and let $\pi_{\theta} \in F_{D}$ a pure strategy defined by $\pi_{\theta}\left(s_{t}\right)=\theta$ for all $s_{t} \in S^{\prime} \backslash\left\{s_{d}\right\}$. From the definition of the TSSP MDP, $C^{\prime}{ }_{s_{t} \theta}=C_{a}$ and $P_{s_{t \theta} g^{*}}=1$ for all $s_{t} \in S^{\prime} \backslash\left\{s_{d}\right\}$, which implies that $V_{\pi_{\theta}}=C_{a}$ and $V_{\pi_{\theta}}<V^{*}$, this contradicts the supposition. In the TSSP MDP, a negative cost can be obtained if and only if a goal state is reached, and the fact that all costs are positives implies that $-C_{g}$ is the lower-bound for the expected total minimum cost.

Proposition 2: If no goal state and no dead end, the expected total minimum cost is finite $\left(\boldsymbol{V}^{*}=\boldsymbol{C}_{\boldsymbol{a}}\right)$ and an $\varepsilon$-optimal pure strategy is $\boldsymbol{\pi}^{*}=\boldsymbol{\pi}_{\boldsymbol{\theta}}$.

Proof: The fact that there exists neither goal state nor dead end state imply that for all $\pi \in F_{D}, \pi \neq \pi_{\theta} s_{t} \in S^{\prime}, a_{t} \in A\left(s_{t}\right)$, we have $C_{s_{t} a_{t} s_{t+1}}^{\prime}>0$, which implies that the expected total cost obtained when the process starts with state $s_{0}$ and using a policy $\pi$ is infinite and so greater than $C_{a}\left(V_{\pi}\left(s_{0}\right)>C_{a}\right)$, which contradict the proposition 1 .

Proposition 3: Let $\boldsymbol{s}_{\mathbf{0}}$ be a given initial state, for $\left(\boldsymbol{C}_{\boldsymbol{a}}<\infty\right)$, if no policy can reach the goal state from $\boldsymbol{s}_{\mathbf{0}}$ then the expected total minimum cost is finite $\left(\boldsymbol{V}^{*}\left(\boldsymbol{s}_{\mathbf{0}}\right)=\boldsymbol{C}_{\boldsymbol{a}}\right)$ and $\pi^{*}\left(s_{0}\right)=\pi_{\theta}\left(s_{0}\right)=\theta$.

Proof: the proof is similar to the last one.

Proposition 4: For $C_{a}=0, C_{g}>0, C_{d}=\frac{C_{g}}{P_{s_{t} a_{t} s_{d}}}\left(C^{\prime}{ }_{{ }_{t} a_{t} s_{d}}=C^{\prime}{ }_{s_{t} a_{t} s_{d}}+\frac{C_{g}}{P_{s_{t} a_{t} s_{d}}}\right.$ if $P_{s_{t} a_{t} s_{d}}>0$ ), an $\varepsilon$-optimal policy for the TSSP MDP surely avoids reaching the aggregated dead end state.

Proof: Let $\pi_{*}\left(V_{\pi^{*}}\right)$ be an optimal pure policy (optimal cost vector) and suppose that the process is in state $s_{t}$ at time $t$ such that $p_{s_{t}} a_{t} s_{d}>0$, and it reaches the aggregated dead end state at time $t+1$, the expected minimum cost when the process starts with state $s_{t}$ is:

$$
V_{\pi^{*}}\left(s_{t}\right)=E_{\pi^{*}}^{s_{t}}\left(C^{\prime}{ }_{s_{t} a_{t}}\right)+\left(\sum_{t^{\prime}=t+1}^{\infty} C^{\prime}{ }_{s_{d} \theta}\right)
$$

The expected cost at time $t$ is given by: 


$$
\begin{aligned}
& E_{\pi^{*}}^{s_{t}}\left(C^{\prime}{ }_{s_{t} a_{t}}\right)=\sum_{s_{t+1} \in S^{\prime} \cup G^{\prime}} P_{s_{t} a_{t} s_{t+1}} \times C^{\prime}{ }_{s_{t} a_{t} s_{t+1}} \\
& E_{\pi^{*}}^{s_{t}}\left(C^{\prime}{ }_{s_{t} a_{t}}\right)=P_{s_{t} a_{t} s_{d}} \times C^{\prime}{ }_{{ }_{s_{t}} a_{t} s_{d}}+X+Y,
\end{aligned}
$$

where the values $X$ and $Y$ are given by:

$$
\begin{aligned}
& X=\sum_{s_{t+1} \in S^{\prime} \backslash s_{\mathrm{d}}} P_{s_{t} a_{t} s_{t+1}} \times C^{\prime}{ }_{s_{t} a_{t} s_{t+1}} \geq 0 \\
& Y=\sum_{\mathrm{g} \in G^{\prime}} \mathrm{P}_{s_{t} a_{t} g} \times \mathrm{C}_{s_{t} a_{t} g}^{\prime}
\end{aligned}
$$

Since the process reaches the aggregated dead end state at time $t+1, a_{t} \neq \theta$, the equation (10) becomes:

$Y=\sum_{g \in G} P_{s_{t} a_{t} g} \times\left(C_{s_{t} a_{t} g}-C_{g}\right)$

The fact that $P_{s_{t} a_{t} g}<1$ (The process does not reach the goal state) and $C_{s_{t} a_{t} g} \geq 0$ for all $g \in G$ implies that $Y>-C_{g}$ and we have: $C^{\prime}{ }_{s_{t} a_{t} s_{d}}=C_{s_{t} a_{t} s_{d}}+C_{d}=C_{s_{t} a_{t} s_{d}}+\frac{C_{g}}{P_{s_{t} a_{t} s_{d}}}$, it follows that:

$P_{s_{t} a_{t} s_{d}} \times C_{s_{t} a_{t} s_{d}}^{\prime}=P_{s_{t} a_{t} s_{d}} \times C_{s_{t} a_{t} s_{d}}+C_{g}$

Therefore,

$E_{\pi^{*}}^{s_{t}}\left(C_{s_{t} a_{t}}^{\prime}\right)=P_{s_{t} a_{t} s_{d}} \times C_{s_{t} a_{t} s_{d}}+C_{g}+X+Y$

Since $X \geq 0$ and $Y>-C_{g}$, it follows that $C_{g}+X+Y>0$ and so: $E_{\pi^{*}}^{S_{t}}\left(C^{\prime}{ }_{s_{t} a_{t}}\right)>$ $C_{a}=0$, which contradicts the proposition $\underline{1}$.

Proposition 5: For $C_{g}=0, C_{a}=E$ and $C_{d}=0$, if the expected total minimum cost for the SSP MDP is greater than $E$, the optimal policy for the TSSP MDP is $\pi^{*}=\pi_{\theta}$.

Proof: The proof follows from the fact that $V_{\pi_{\theta}}^{*}=C_{a}=E$ and no optimal cost vector can be greater that $E$.

Proposition 6: For $C_{g}=E, C_{a}=0$ and $C_{d}=0$, if the expected total minimum cost for the SSP MDP is greater than $E$, the $\varepsilon$-optimal policy for the TSSP MDP is $\pi^{*}=\pi_{\theta}$.

Proof: Let $V_{S S P}^{*}\left(V_{T S S P}^{*}\right)$ be the expected minimum cost for the SSP MDP (TSSP MDP), it is evident that $V_{T S S P}^{*}=V_{S S P}^{*}-E$, consequently, if $V_{S S P}^{*}>C_{g}$ implies $V_{T S S P}^{*}>0$ and we have $V_{\pi_{\theta}}^{*}=C_{a}=0$ then $\pi^{*}=\pi_{\theta}$.

Proposition 7: If $\left(C_{a}=\infty, C_{d} \simeq \infty, C_{g} \simeq \infty\right)$, an optimal policy generally maximizes the probability of reaching the goal and minimizes the expected cost.

Proof: Let $\pi_{1}\left(\pi_{2}\right)$ be a strategy that reaches the goal state with probability $P_{1}\left(P_{2}\right)$ such that $P_{2}>P_{1}$ and $V_{\pi_{1}}\left(V_{\pi_{2}}\right)$ be the expected cost vector obtained with $\pi_{1}\left(\pi_{2}\right)$.

For $C_{d} \simeq \infty$ and $C_{g} \simeq \infty$, we have: $V_{\pi_{1}} \simeq-P_{1} \times C_{g}+\left(1-P_{1}\right) \times C_{d}$ and $V_{\pi_{2}} \simeq-P_{2} \times$ $C_{g}+\left(1-P_{2}\right) \times C_{d}$. We have $P_{2}>P_{1}$ then $-P_{1} \times C_{g}>-P_{2} \times C_{g}$ and $\left(1-P_{1}\right) \times C_{d}>$ $\left(1-P_{2}\right) \times C_{d}$, which implies $V_{\pi_{1}}>V_{\pi_{2}}$.

Note that $\boldsymbol{C}_{\boldsymbol{d}} \simeq \infty$ and $\boldsymbol{C}_{\boldsymbol{g}} \simeq \infty$ mean that $\boldsymbol{C}_{\boldsymbol{d}}$ and $\boldsymbol{C}_{\boldsymbol{g}}$ are considerably very large. 
Lemma: For any initial cost vector $\mathrm{V}^{0} \geq \mathrm{V}^{*}$, the PGSVI algorithm converges monotonically to an $\varepsilon$-optimal solution in a finite number of iterations.

Proof: The proof follows from the last propositions and the existence of absorbing states (goal states or abstract goal) [4], [14].

\section{TOPOLOGICAL SOLVER}

Hierarchical or Topological method is one of the most solutions used by several researchers to alleviate the burden of high dimensionality [15]. It was introduced by Ross and Varadarajan [16] for constrained limiting average MDP and used by Abbad and Boustique [17]; Abbad and Daoui [18]; Daoui and Abbad [19], [20]; Dai and Goldsmith [21] and Larach et al., [22] in several categories of MDPs. They consist on decomposing the original MDP into smaller problems called restricted MDPs, which are solved according to some order (levels) and combined to obtain a global solution. Dai P and Goldsmith J [21] present a topological VI algorithm based on Kosaraju's decomposition algorithm into SCCs [23], Larach et al., [22] present a hierarchical VI algorithm for discounted MDPs using a new definition of the restricted MDPs and a new decomposition algorithm into levels; in each level, independent restricted MDPs can be solved in parallel. However, these hierarchical algorithms are not efficient if the original MDP is communicating. Consequently, the authors present another decomposition technique that is efficient for several domains of MDPs with goal states. It is based on the decomposition of the state space into levels corresponding to the topological sort. The overall heuristic solution obtained is used as an initial cost vector in the PGSVI algorithm, which permits to reduce the number of iterations needed to attain an optimal or $\varepsilon$-optimal solution.

\subsection{DECOMPOSITION TECHNIQUE}

Let $\mathrm{Gr}=(\mathrm{S}, \mathrm{U})$ be the associated graph to the original TSSP MDP, where the state space $S$ represents the set of nodes and $U=\left\{(i, j) \in S^{2}: \exists a \in A(i), P_{\text {iaj }}>0\right\}$ is the set of directed arcs and let $\mathrm{G}_{\mathrm{g}}$ be the set of goal states. The level 0 contains all goal states, the level 1 contains all states that can reach any goal state in one transition; the level 2 contains all states that can reach the level 1 in one transition, etc. Levels are constructed as follows: $L_{0}=\mathrm{G}_{\mathrm{g}}, \quad L_{l}=\left\{i \in S: \exists a \in A(i), \exists j \in L_{0}, P_{i a j}>0\right\}, \quad L_{k}=\{i \in S: \exists a \in$ $\left.A(i), \exists j \in L_{k-1}, P_{i a j}>0\right\}$, (see example in Fig. 2).

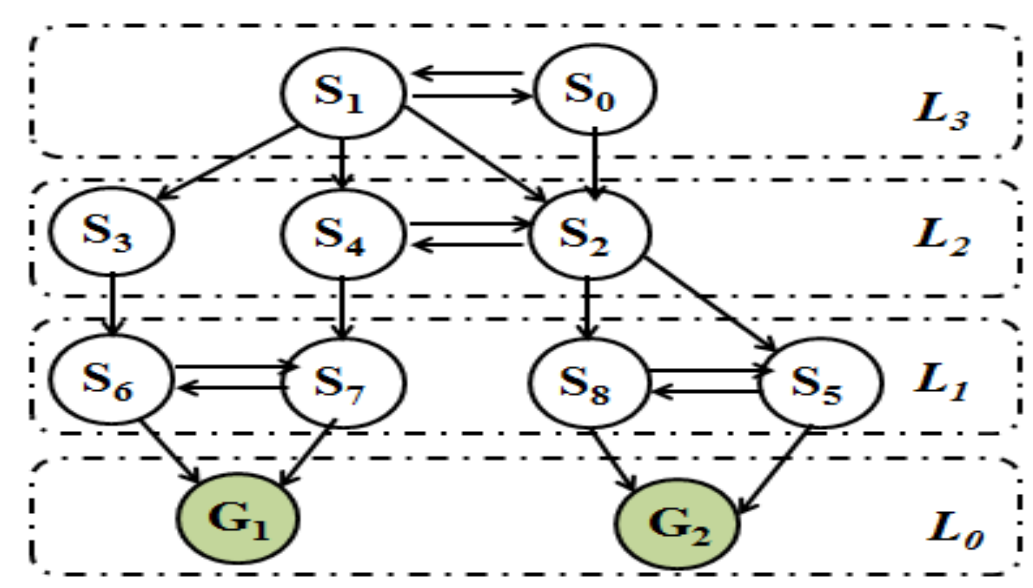

Fig. 2 Example of an associated SSP MDP graph decomposed into levels 


\subsection{DECOMPOSITION INTO LEVELS}

The Breadth-First Search (BFD) algorithm can be used to construct levels by starting analysis from goal states and searching for non-visited states contained in the next level until all reachable states are visited. The authors use the following BFS algorithm (Algorithm 2), where $\Gamma^{-}(i)=\left\{j \in S: \exists a \in A(j), P_{j a i}>0\right\}$ designs the predecessors set of $i$.

After initializing the visited parameter (false for all states and true for goal states) (lines 1 and 2, algorithm 2), the search of levels starts with goal states (lines 3 and 4, algorithm 2) and is terminated after all states are visited or no predecessor is found (line 5, algorithm 2). The next level is initialized by $\emptyset$ (line 6 , algorithm 2) and for all non-visited states in the predecessors set of the current level (lines 7-9, algorithm 2), the predecessor is added to the next level (line 10, algorithm 2) and marked as visited (line 11, algorithm 2). The algorithm 2 runs at $O\left(|S|\left|\Gamma^{-}\right|\right.$) where $|S|$ is the number of states and $\left|\Gamma^{-}\right|$is the average number of predecessors per state.

Algorithm 2: Decomposition into levels
BFS_levels (In: S, G; Out: L)
//Initialisation
1: For each i $\in$ S Do i. visited $\leftarrow$ False;
2: For each $i \in G$ Do i. visited $\leftarrow$ True;
3: $\mathbf{L}_{\mathbf{0}} \leftarrow \mathrm{G} / /$ The set of goals states
4: $\mathrm{k} \leftarrow 0$; //Level identifier

\section{//Levels search until non-unvisited state founded}

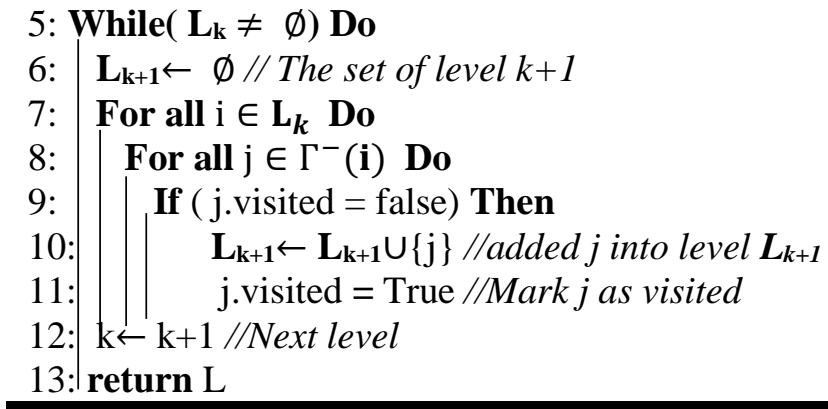

\subsection{RESTRICTED SSP MDPs}

Using the decomposition adopted above, the authors define a restricted TSSP $\mathrm{MDP}_{\mathrm{k}}$ associated to each level $k(k=1,2 \ldots)$ as follows:

- State space: $\quad S^{k}=L_{k}$

- Action space: $\mathrm{A}^{k}(i)=A^{\prime}(i)$, for alli $\in \mathrm{S}^{k}$.

- Transition: For all $i \in \mathrm{S}^{k}, P^{k}{ }_{i a j}=P^{\prime}{ }_{i a j}$ if $j \in\left\{\mathrm{S}^{k-1} \cup S^{k}\right\}$ and $P^{k}{ }_{i a j}=0$ otherwise.

- Cost function: For all $i \in \mathrm{S}^{k}, \quad \mathrm{C}^{k}{ }_{i a j}=C^{\prime}{ }_{i a j}+P^{\prime}{ }_{i a j} V^{*}(j)$ if $j \in \mathrm{S}^{k-1}$ and $\mathrm{C}^{k}{ }_{i a j}=C^{\prime}{ }_{i a j}$ otherwise, where $V^{*}(j)$ is the optimal value calculated in the previous level $L_{k-1}$.

The restricted SSP $\operatorname{MDP}_{\mathrm{k}}(\mathrm{k}=1,2 \ldots)$ are solved in ascending order of levels. If $\mathrm{V}_{k}^{H}$ designs the solution obtained for the restricted SSP $\mathrm{MDP}_{\mathrm{k}}$, the combined solution $V^{H}=$ $\mathrm{U}_{k} \mathrm{~V}_{k}^{H}$ gives an admissible heuristic solution for the original problem. 
Remark 1: Note that, if a level $L_{k}$ contains $p$ non-communicating classes (e.g.,

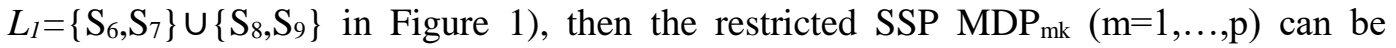
solved in parallel.

\subsection{TOPOLOGICAL PGSVI SOLVER}

After the decomposition of the state space into levels and the construction of the restricted SSP MDPs, the heuristic solution $\mathrm{V}^{\mathrm{H}}$ is obtained by applying the PGSVI algorithm (Algorithm 1) to each restricted SSP MDP in ascending order of levels, which is usually so close to the $\varepsilon$-optimal solution and thus can be used as an initial cost vector in the PGSVI algorithm. Consequently an $\varepsilon$-optimal solution is obtained in a few number of iterations (see Table I).

Algorithm 3: TPGSVI Algorithm

TPGSVI(In: SSP MDP: S, P, A, C,G, $\varepsilon ; E, C_{g}, C_{d}, C_{a} ;$ Out: $\left.\mathrm{V}^{*}, \pi^{*}\right)$

1. $\mathrm{L} \leftarrow$ BFS_levels $(\mathrm{S}, \mathrm{G}) / /$ Construct levels using algorithm 2

2. For each level $\mathrm{L}_{\mathrm{k}}, \mathrm{k}=1, \ldots \mathrm{N}_{\mathrm{l}}$ Do //Construct the restricted MDPs

TSSP_MDP ${ }_{\mathrm{k}} \leftarrow$ Restricted_MDP(TSSP_MDP, $\left.\mathrm{L}_{\mathrm{k}}\right)$

3. For each level $\mathrm{L}_{\mathrm{k}}, \mathrm{k}=1, \ldots \mathrm{N}_{\mathrm{l}}$ Do // Solve the restricted $M D P_{k}, k=1, \ldots N_{l}$

$\left(\mathrm{V}_{k}^{H}, \pi_{k}^{H}\right) \leftarrow$ PGSVI$\left(\right.$ TSSP_MDP,$\left.V^{0}=0\right)$

4. $V^{H}=\mathrm{U}_{k} \mathrm{~V}_{k}^{H} / /$ Combine the solutions obtained

5. $\left(\mathrm{V}^{*}, \pi^{*}\right) \leftarrow$ PGSVI(TSSP_MDP, $\left.V^{H}\right) / /$ Use $V^{H}$ as initial vector

6. Return $V^{*}, \pi^{*}$

Theorem: The algorithm 3 works correctly and converges to an $\varepsilon$-optimal solution in a finite number of iterations.

Proof: Since each restricted TSSP MDP gives the heuristic cost vector $V_{k}^{H} \geq V^{*}$, the lemma proves the convergence of the TPGSVI algorithm.

Remark 2: Finding the set of initial states denoted by $\mathrm{S}_{1}$ from where the goal can be reached with $100 \%$ probability is so important in some dramatically situations. One solution consists on using the MAXPROB algorithm [12] to find the maximum PROB vector $\mathrm{P}^{*}$ and in this case $\mathrm{S}_{1}=\left\{i \in S: P^{*}(i)=1\right\}$. Another solution consists on using the Breadth-First Search (BFD) algorithm by starting the analysis from dead end states and searching the following levels: $L_{0}=\mathrm{S}_{\mathrm{d}}, L_{l}=\left\{i \in S: \forall a \in A(i), \exists j \in L_{0}, P_{i a j}>0\right\} \ldots$ $L_{k}=\left\{i \in S: \forall a \in A(i), \exists j \in L_{k-1}, P_{i a j}>0\right\}$ and in this case $S_{1}=S \backslash\left\{L_{0} \cup L_{1} \cup \ldots \cup L_{k}\right\}$.

\section{SIMULATION AND EXPERIMENTAL RESULTS}

To show the advantages of the proposed TSSP MDP and the topological solver, we have used an example of robotic navigation, in a hexagonal grid's environment and simulated it using java-applet language. The computational performance of the proposed iterative solver is compared with PGSVI and VI algorithms using Intel(R) Core(TM) i5$6500 \mathrm{CPU} @ 3,20 \mathrm{GHz}, \mathrm{C}++$ implementation Windows 7 operating system (64 bits).

\subsection{SSP MDP MODEL FOR ROBOT NAVIGATION}

We consider a robot navigation problem, in a hexagonal grid's environment containing obstacles and buried mines considered as dead end states, the agent must avoid all obstacles and buried mines and find an optimal path to navigate from any given initial state to the nearest goal state with minimum cost of energy. The problem is formulated as an SSP MDP. Each grid cell is free, obstacle, buried mine 
or goal region. The state space is therefore a set of grids and can be represented by a matrix of values; each grid cell has an associated value-stating, obstacle (1), free (0), goal state (2) or buried mine state (3). We assume that the robot is controlled through six actions: the six directions in the hexagonal grids. Actions that can directly move the robot to an obstacle or to a buried mine state are eliminated.

We assume that the transition to a free or goal state is characterized by a cost of energy consumed (denoted by $\boldsymbol{e}$ ) proportional to the distance travelled.

The transition function defines the uncertainty due to the effects of actions, it is very difficult to model and can be determined by the reinforcement learning process. For a simple case, we use the transition function defined in Figure 3, the agent transits to the desired state with $80 \%$ probability and to one of the two near states with $10 \%$ probability, we assume that when the transition probability to an obstacle is different than zero, the robot's position remains the same (Figure 3, right) and the cost is $C_{i a i}=\boldsymbol{e}$.

Remark 3: By using the previous model in a hexagonal grid's environment, the number of actions per state and the average number of state-action successors are very small (seven actions per state and three successors per state-action) and they can be considered as constant values. The PGSVI algorithm is therefore linear per iteration $(O(|\mathrm{~S}|) /$ iteration $)$.

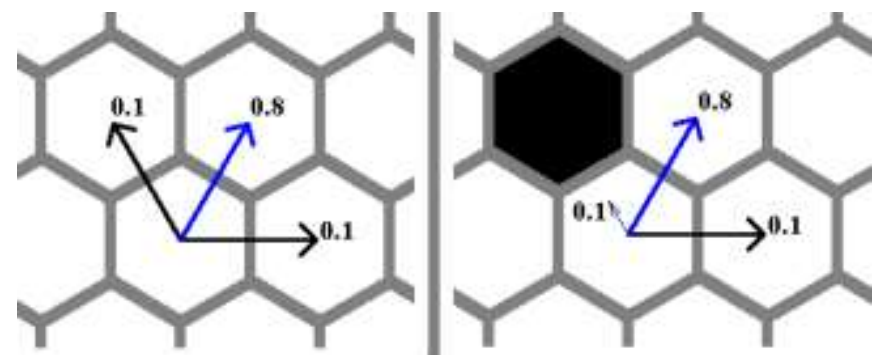

Fig. 3 Example of the transition probabilities

\subsection{UNREACHABLE GOAL STATE AND INSUFFICIENT ENERGY CASES}

Figure 4 shows two examples of optimal policies indicated by arrows and optimal cost vectors in an environment containing obstacles (black cell, Obs) and one goal state (green cell, $\mathrm{G})$ and using the parameters $\mathrm{e}=1, \mathrm{E}=10 \mathrm{e}, \varepsilon=10^{-6},\left(\mathrm{C}_{\mathrm{g}}=\mathrm{E}, \mathrm{C}_{\mathrm{a}}=0, \mathrm{C}_{\mathrm{d}}=0\right.$, in Figure 4, left), and the parameters $\mathrm{e}=1, \mathrm{E}=10 \mathrm{e}, \varepsilon=10^{-6},\left(\mathrm{C}_{\mathrm{g}}=0, \mathrm{C}_{\mathrm{a}}=\mathrm{E}, \mathrm{C}_{\mathrm{d}}=0\right.$, in Figure 4 , right $)$.

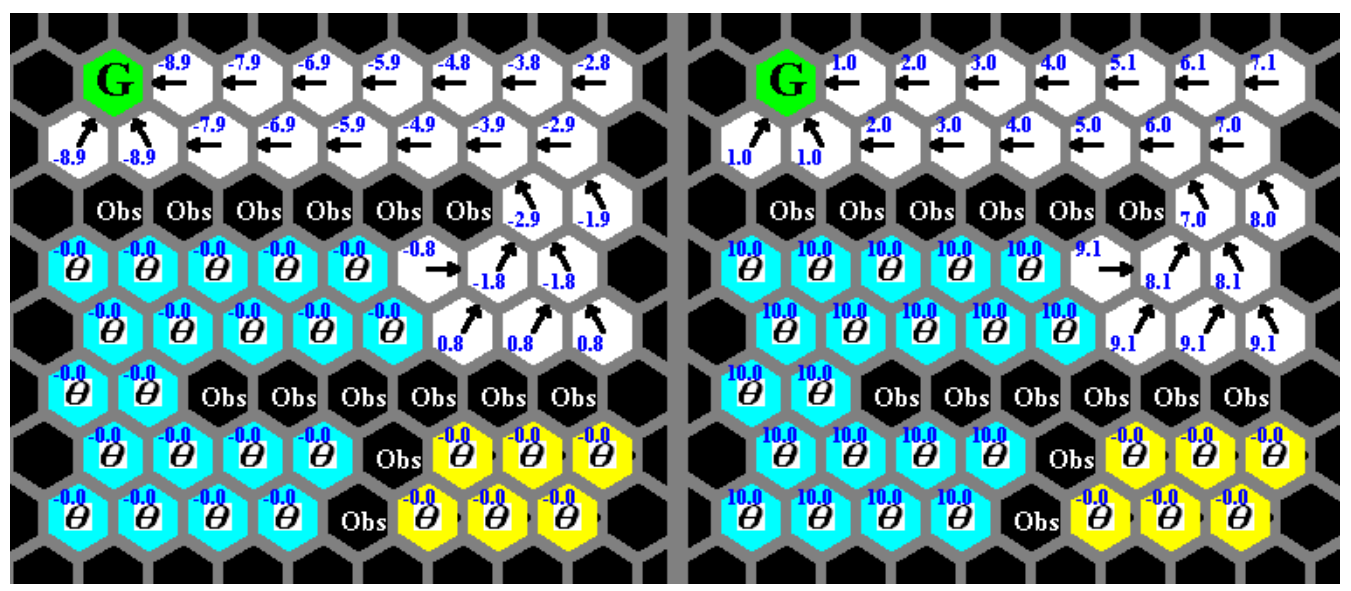

Fig. 4 Example of an $\varepsilon$-optimal strategy and $\varepsilon$-optimal cost vector using the parameters: $\mathrm{e}=1, \mathrm{E}=10 \mathrm{e}, \varepsilon=10^{-6}$, (left: $\left.\mathrm{C}_{\mathrm{g}}=\mathrm{E}, \mathrm{C}_{\mathrm{a}}=0, \mathrm{C}_{\mathrm{d}}=0\right)$, (right: $\left.\mathrm{C}_{\mathrm{g}}=0, \mathrm{C}_{\mathrm{a}}=\mathrm{E}, \mathrm{C}_{\mathrm{d}}=0\right)$ 
As it can be seen, the two strategies are equivalents. When no policy can reach the goal (unreachable goal state case), the agent cannot achieve the target (yellow cells) and an optimal action is $\theta$ (proposition 2) and if the expected minimum total cost needed to reach the goal is greater than the total cost supported (cyan cells, insufficient resource case), the optimal action is $\theta$ (propositions 3 and 4 ).

Remark 4: If the above transformation is not used, VI or PGSVI algorithms diverge for the example presented in Figure 4, because the minimum cost vector will be infinite for the states from which the agent cannot reach the goal.

\subsection{AVOIDING REACHING THE DEAD END STATE}

Figure 5 shows an example of optimal policies using the parameters: $\mathrm{e}=1, \mathrm{E}=20 \mathrm{e}$, $\varepsilon=10^{-6}$ (Left: $C_{d}=200, \mathrm{C}_{\mathrm{a}}=0, \mathrm{C}_{\mathrm{g}}=\mathrm{E}$ ) and (right: $C_{d}=200, \mathrm{C}_{\mathrm{a}}=\mathrm{E}, \mathrm{C}_{\mathrm{g}}=0$ ). As it can be seen, for the initial states (cyan cells) where no policy can surely avoid reaching the dead end state, the optimal action is $\theta$, and for the other states, the agent surely avoids reaching the dead end state but with no guarantee to reach the goal for some cases (see Figure 6, left).

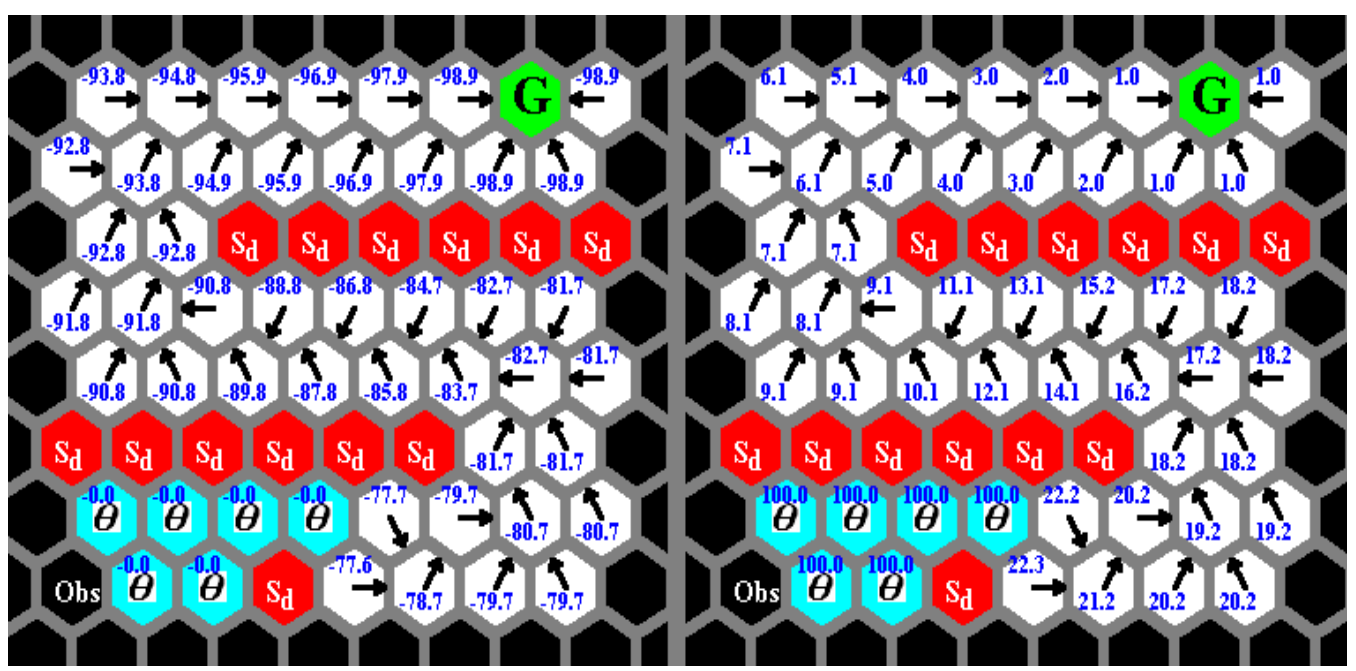

Fig. 5 Example of an E-optimal policy indicated by arrows and cost vector values in a random environment containing dead end states (red cells, $S_{\mathrm{d}}$ ) and using the parameters: $\mathrm{e}=1, \mathrm{E}=100 \mathrm{e}, \varepsilon=10^{-6}$, (Left: $\mathrm{C}_{\mathrm{d}}=200, C_{a}=0, C_{g}=E$ ) and (right: $\mathrm{C}_{\mathrm{d}}=200, C_{a}=E, C_{g}=0$ ).

\subsection{REACHING THE GOAL WITH 100\% PROBABILITY}

The proposed technique to find an optimal policy that surely avoids reaching the dead end state cannot guarantee to reach the goal for all initial states with an optimal action different than $\theta$. 


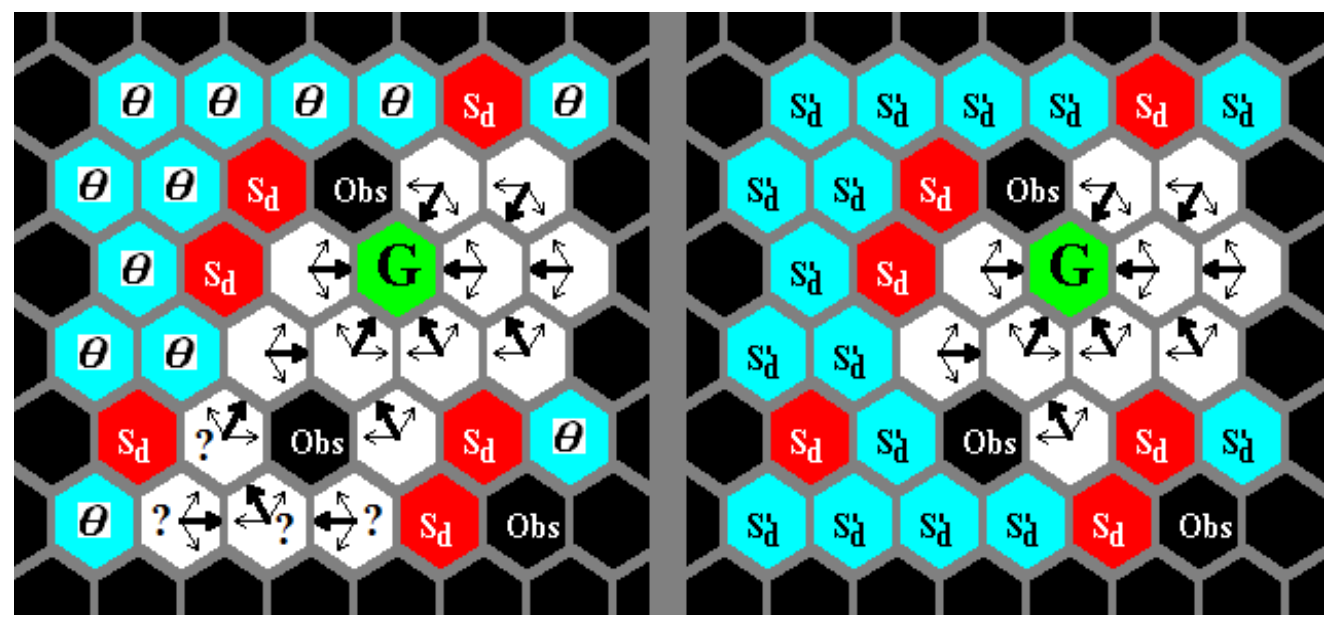

Fig. 6 Example of an optimal policy that surely avoids reaching the dead end or/and surely reaches the goal

In Figure 6 (left), if the process starts in some states indicated by “?”, it can transit to another state with an optimal action $\theta$ and reside in it. To find the set of all initial states that guarantees the goal reachability, we can apply the technique indicated in Remark 2. Figure 6 (right) shows the obtained $\varepsilon$-optimal policy. As it can be seen, all states from where no policy can surely moves the process to the goal are considered as dead ends.

\subsection{MAXPROB-MINCOST EXAMPLE}

Figure 7 (left) shows an $\varepsilon$-optimal policy obtained by the MAXPROB-MINCOST algorithm used by [12] compared to an $\varepsilon$-optimal policy obtained using the proposition 5 . As it can be seen, we obtain the same policy that maximizes the probability to reach the goal and minimizes the expected cost.

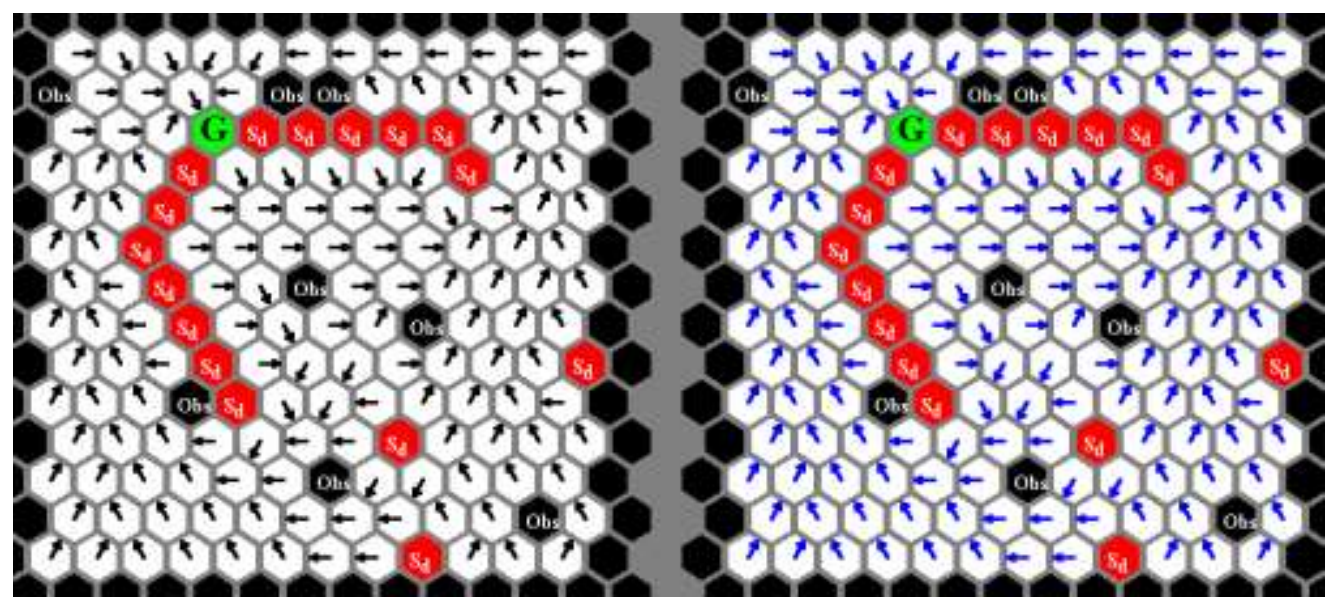

Fig. 7 Example of an optimal policy obtained by the MAXPROB-MINCOST algorithm used by [12] (left) and an optimal policy obtained by the proposed MAXPROB-

MINCOST method (proposition 7) (right)

\subsection{PERFORMANCE OF THE TPGSVI ALGORITHM}

After initializing the PGSVI algorithm by the heuristic solution obtained with the restricted TSSP MDPs based on the decomposition technique, an $\varepsilon$-optimal solution is attained after a small number of iterations (see Table 1). 
Table I. Number of iterations before convergence and after using the heuristic solution in the PGSVI algorithm

\begin{tabular}{cc}
\hline Number of states & Number of iterations \\
\hline $10^{5}$ & 4 \\
$4 \times 10^{5}$ & 8 \\
$8 \times 10^{5}$ & 11 \\
$12 \times 10^{5}$ & 14 \\
\hline
\end{tabular}

Figure 8 shows the average computation time of PGSVI, VI and TPGSVI algorithms under random models in a hexagonal grid's environment with ten goals states, $5 \%$ dead end states and 5\% obstacles. The results show that PGSVI almost outperforms VI since it reduces the number of iterations and the TPGSVI algorithm is more efficient in this hexagonal grid's environment.

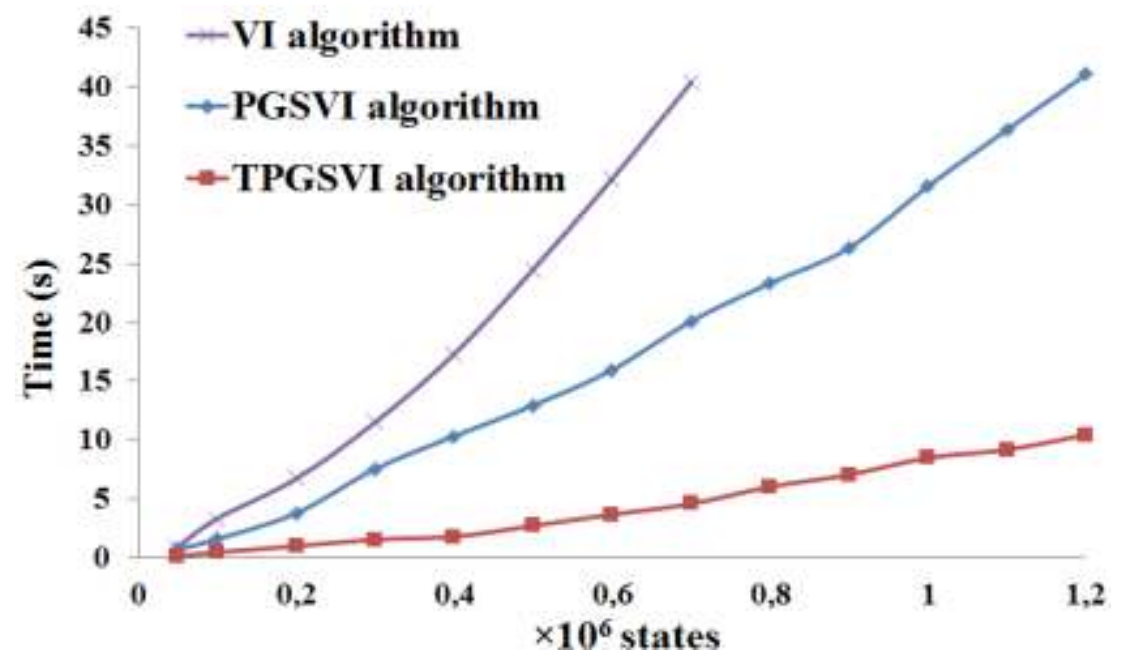

Fig. 8 Performance of the TPGSVI algorithm compared with PGSVI and VI algorithms

\section{DISCUSSION}

The approaches proposed by Bonet and Geffner [6]-[8] that find a heuristic solution and cannot guarantee the convergence of the iterative algorithms with dead ends can be applied to this proposed TSSP MDP in which the convergence is assured by two techniques: (i) the abstract goal state added, (ii) the topological solver proposed (the decomposition technique into levels cannot contain any dead end state). An approach that resembles FIND-and-REVISE heuristic search framework [8], named Accelerated Hierarchical VI algorithm proposed by [22] and based on the decomposition of the state space into Strongly Connected Component and there belonging levels, can be used in the undiscounted MDP to find an ع-optimal solution.

The proposed TSSP offers several choices according to the values of the added parameters, which is an important advantage over the approaches proposed by Weld et al [10], Teichteil-Konigsbuch [11] and Kolobov et al., [12]. Also the energy constraint, which is not treated by these works, is easily solved with the added parameters. The MaxProb-MinCost criterion can be generally solved using the proposition 7 without using two iterative algorithms. The proposed TPGVI algorithm offers the possibility to accelerate the convergence towards the optimal solution in several domains. Finally, the TSSP can be extended to the case where the energy can be increased in some states. 


\section{CONSLUSION}

In this paper, we have proposed a new Transformed SSP MDP depending on some added parameters that have main advantages: (1) the convergence of the classical iterative algorithms with dead ends problem; (2) the energy-reachability problem is easily solved; (3) the dead end state is surely avoided; (4) the goal state is surely reached with minimum cost or the agent stays up; (5) the agent can stay up, in a dynamic SSP, until its probability to reach the goal state is equal to 1 . We have also presented a MAXPROBMINCOST algorithm that maximizes the probability of reaching the goal and minimizes the expected cost without using two iterative algorithms. Finally, we have presented a topological solver based on a decomposition of the original SSP MDP into smaller problems solved in ascending order of levels and combined to give a heuristic cost vector used as initial point in the PGSVI algorithm, which permit to accelerate the solver in several types of SSP MDP. The experimental results in the robot navigation example show that the proposed solver outperforms the standard solvers of SSP MDPs. In future work, we try to apply the proposed approach in a dynamic SSP problem and extended it to the case where the energy levels can increase in some transitions. Also, we try to solve this TSSP with finite horizon MDP, which can be more efficient.

\section{REFERENCES}

[1] D. J. White, "A survey of applications of Markov decision processes", Journal of the operational research society, vol. 44, no 11, (1993): 1073-1096.

[2] A. Larach, C. Daoui, and M. Baslam, "A Markov Decision Model for Area Coverage in Autonomous Demining Robot", International Journal of Informatics and Communication Technology (IJ-ICT), vol. 6, no 2, (2017): 105-116.

[3] A. R. Sfar, E. Natalizio, Y. Challal, "A Markov game privacy preserving model in retail applications", In Selected Topics in Mobile and Wireless Networking (MoWNeT), 2017 International Conference on IEEE, (2017): 1-8.

[4] M. L. Puterman, "Markov decision processes: discrete stochastic dynamic programming", John Wiley \& Sons, (2014).

[5] D. P. Bertsekas, and J. N. Tsitsiklis, "An analysis of stochastic shortest path problems", Mathematics of Operations Research, vol. 16, no 3, (1991): 580-595.

[6] B. Bonet, and H. Geffner, "Solving stochastic shortest-path problems with RTDP", Universidad Simon Bolivar, Tech. Rep., (2002).

[7] B. Bonet and H. Geffner, "Labeled RTDP: Improving the Convergence of Real-Time Dynamic Programming”, In: ICAPS. (2003): 12-21.

[8] B. Bonet, and H. Geffner, "Faster heuristic search algorithms for planning with uncertainty and full feedback", In: IJCAI. (2003): 1233-1238.

[9] A. Kolobov, D. S. Weld, H. Geffner, "Heuristic search for generalized stochastic shortest path MDPs", In: Proceedings of the Twenty-First International Conference on International Conference on Automated Planning and Scheduling, AAAI Press, (2011): 130-137.

[10] D. S. Weld and A. Kolobov, "Stochastic Shortest Path MDPs with Dead Ends", HSDIP, (2012): 78.

[11] F. Teichteil-Konigsbuch, "Stochastic safest and shortest path problems", Proceedings of the TwentySixth AAAI Conference on Artificial Intelligence, AAAI Press, (2012): 1825-1831.

[12] A. Kolobov, D. S. Weld, "A theory of goal-oriented MDPs with dead ends", arXiv preprint arXiv: 1210.4875, (2012).

[13] F. Trevizan, F. Teichteil-Konigsbuch, and S. Thiebaux, "Efficient solutions for stochastic shortest path problems with dead ends", In: Proceedings of the Thirty-Third Conference on Uncertainty in Artificial Intelligence, UAI, (2017): 11-15.

[14] R. E. Bellman, "Dynamic Programming", Princeton University Press. Princeton, NJ, (1957).

[15] M. L. Littman, T. L. Dean, and L. P. Kaelbling, "On the complexity of solving Markov decision problems", In: Proceedings of the Eleventh conference on Uncertainty in artificial intelligence. Morgan Kaufmann Publishers Inc., (1995): 394-402.

[16] K. W. Ross, and R. Varadrajan, "Multi-chain Markov decision processes with a sample path constraint: A decomposition approach", Mathematics of Operations Research, vol. 16, no 1, (1991): 195-207.

[17] M. Abbad, and H. Boustique, "A decomposition algorithm for limiting average Markov decision problems", Operations Research Letters, vol. 31, no 6, (2003): 473-476.

[18] M. Abbad and C. Daoui, "Hierarchical algorithms for discounted and weighted Markov decision processes", Mathematical Methods of Operations Research, vol. 58, no 2, (2003): 237-245. 
[19] C. Daoui, and M. Abbad, "One some algorithms for limiting average Markov decision processes", Operations Research Letters, vol. 35, no 2, (2007): 261-266.

[20] C. Daoui, M. Abbad and M. Tkiouat, "Exact decomposition approaches for Markov decision processes: A survey", Advances in Operations Research, vol. 2010, (2010).

[21] P. Dai, D. S. Weld and J. Goldsmith, "Topological value iteration algorithms", Journal of Artificial Intelligence Research, vol. 42, (2011): 181-209.

[22] A. Larach, S. Chafik and C. Daoui, "Accelerated decomposition techniques for large discounted Markov decision processes", Journal of Industrial Engineering International, vol. 13, no 4, (2017): 417426.

[23] M. Sharir, "A Strong-connectivity Algorithm and its Applications in Data Flow analysis”, Computers \& Mathematics with Applications, 7(1), (1981): 67-72. 
International Journal of Advanced Science and Technology Vol.129 (2019) 